\title{
Ergodicity of the implicit midpoint rule for nonexpansive mappings
}

Hong-Kun $\mathrm{Xu}^{1,2^{*}}$, Maryam A Alghamdi ${ }^{3}$ and Naseer Shahzad ${ }^{2}$

"Correspondence:

hkxuukzn@yahoo.com

${ }^{1}$ Department of Mathematics,

School of Science, Hangzhou Dianzi

University, Hangzhou, Zhejiang

310018, China

${ }^{2}$ Department of Mathematics, King

Abdulaziz University, P.O. Box 80203,

Jeddah, 21589, Saudi Arabia

Full list of author information is

available at the end of the article

\begin{abstract}
We prove a mean ergodic theorem for the implicit midpoint rule for nonexpansive mappings in a Hilbert space. We obtain weak convergence for the general case and strong convergence for certain special cases.
\end{abstract}

MSC: 47J25; 47N20; 34G20; 65J15

Keywords: ergodic; implicit midpoint rule; nonexpansive mapping; projection; Hilbert space

\section{Introduction}

The first mean ergodic theorem for nonlinear noncompact operators was proved by Baillon [1]. Let $C$ be a closed convex subset of a Hilbert space $H$ and let $T: C \rightarrow C$ be a nonexpansive mapping (i.e., $\|T x-T y\| \leq\|x-y\|$ for all $x, y \in C$ ) with fixed points. Then, for each $x \in C$, the Cesàro means

$$
S_{n}(x):=\frac{1}{n} \sum_{k=0}^{n-1} T^{k} x, \quad n \geq 1,
$$

converge weakly to a fixed point of $T$. This mean ergodic theorem was extended by Bruck [2] to the setting of Banach spaces that are uniformly convex and have a Fréchet differentiable norm. Baillon and Clement [3] also investigated ergodicity of the nonlinear Volterra integral equations in Hilbert spaces.

It is quite natural to consider ergodic convergence of iterative algorithms in the case where the sequences generated by the algorithms either are not guaranteed to converge or not convergent at all. For instance, the double-backward method of Passty [4] generates a sequence $\left\{x_{n}\right\}$ in the recursive manner:

$$
x_{n+1}=\left(J_{B}^{\lambda_{n+1}} \circ J_{A}^{\lambda_{n+1}}\right) x_{n}, \quad n \geq 0,
$$

where $A$ and $B$ are maximal monotone operators in a Hilbert space such that $A+B$ is also maximal monotone and the inclusion $0 \in(A+B) x$ is solvable, and $J_{A}^{\lambda}$ and $J_{B}^{\lambda}$ are the resolvents of $A$ and $B$, respectively, that is, $J_{A}^{\lambda}=(I+\lambda A)^{-1}$ and $J_{B}^{\lambda}=(I+\lambda B)^{-1}$. It is well known [5] that the sequence $\left\{x_{n}\right\}$ generated by the double-backward method (1.1) fails to converge weakly, in general. However, Passty [4] showed that if the sequence of parameters, $\left\{\lambda_{n}\right\}$, is

C2015 Xu et al.; licensee Springer. This is an Open Access article distributed under the terms of the Creative Commons Attribution License (http://creativecommons.org/licenses/by/2.0), which permits unrestricted use, distribution, and reproduction in any medium, provided the original work is properly cited. 
in $\ell^{2} \backslash \ell^{1}$, then the averages

$$
z_{n}:=\frac{\sum_{i=1}^{n} \lambda_{i} x_{i}}{\sum_{i=1}^{n} \lambda_{i}}, \quad n=1,2, \ldots
$$

converge weakly to a solution to the inclusion $0 \in(A+B) x$.

The implicit midpoint rule (IMR) for nonexpansive mappings in a Hilbert space $H$, inspired by the IMR for ordinary differential equations [6-12], was introduced in [13]. This rule generates a sequence $\left\{x_{n}\right\}$ via the semi-implicit procedure:

$$
x_{n+1}=\left(1-t_{n}\right) x_{n}+t_{n} T\left(\frac{x_{n}+x_{n+1}}{2}\right), \quad n \geq 0,
$$

where the initial guess $x_{0} \in C$ is arbitrarily chosen, $t_{n} \in(0,1)$ for all $n$, and $T: C \rightarrow C$ is a nonexpansive mapping with fixed points.

The IMR (1.3) is proved to converge weakly [13] in the Hilbert space setting provided the sequence $\left\{t_{n}\right\}$ satisfies the two conditions:

(C1) $t_{n+1}^{2} \leq a t_{n}$ for all $n \geq 0$ and some $a>0$, and

(C2) $\liminf _{n \rightarrow \infty} t_{n}>0$.

However, this algorithm may fail to converge weakly without the assumption $(\mathrm{C} 2)$. We therefore turn our attention to the ergodic convergence of the algorithm. We will show that for any sequence $\left\{t_{n}\right\}$ in the interval $(0,1)$, the mean averages $\left\{z_{n}\right\}$ as defined by (1.2) will always converge weakly to a fixed point of $T$ as long as $\left\{x_{n}\right\}$ is an approximate fixed point of $T$ (i.e., $\left\|x_{n}-T x_{n}\right\| \rightarrow 0$ ). We will also show that under certain additional conditions the means $\left\{z_{n}\right\}$ can converge in norm to a fixed point of $T$. This paper is organized as follows. In the next section we introduce the concept of nearest point projections and properties of nonexpansive mappings. The main results of this paper (i.e., weak and strong ergodicity of the IMR (1.3)) are presented in Section 3.

\section{Preliminaries}

Let $C$ be a nonempty closed convex subset of a Hilbert space $H$. Recall that the nearest point projection from $H$ to $C, P_{C}$, is defined by

$$
P_{C} x:=\arg \min _{y \in C}\|x-y\|^{2}, \quad x \in H .
$$

We need the following characterization of projections.

Lemma 2.1 Let $C$ be a nonempty closed convex subset of a Hilbert space $H$. Given $x \in H$ and $z \in C$, then $z=P_{C} x$ if and only if any one of the following properties is satisfied:

(i) $\|x-z\| \leq\|x-y\|$ for all $y \in C$;

(ii) $\langle x-z, y-z\rangle \leq 0$ for all $y \in C$;

(iii) $\|x-z\|^{2} \leq\|x-y\|^{2}-\|z-y\|^{2}$ for all $y \in C$.

Recall that a mapping $T: C \rightarrow C$ is said to be nonexpansive if

$$
\|T x-T y\| \leq\|x-y\|, \quad x, y \in C .
$$


A point $x \in C$ such that $T x=x$ is said to be a fixed point of $T$. The set of all fixed points of $T$ is denoted by Fix $(T)$, namely,

$$
\operatorname{Fix}(T)=\{x \in C: T x=x\} .
$$

In the rest of this paper we always assume $\operatorname{Fix}(T) \neq \emptyset$.

We need the demiclosedness principle of nonexpansive mappings as described below.

Lemma 2.2 [14] Let $C$ be a closed convex subset of a Hilbert space $H$ and let $T: C \rightarrow C$ be a nonexpansive mapping. Then the mapping $I-T$ is demiclosed in the sense that, for any sequence $\left\{x_{n}\right\}$ of $C$, the following implication holds:

$$
x_{n} \rightarrow x \text { weakly and }(I-T) x_{n} \rightarrow 0 \text { in norm } \quad \Longrightarrow \quad(I-T) x=0 .
$$

Next we need the following lemma (not hard to prove).

Lemma 2.3 [15] For each integer $n \geq 2, \lambda_{1} \geq 0, \ldots, \lambda_{n} \geq 0$ such that $\lambda_{1}+\cdots+\lambda_{n}=1$, points $u_{1}, \ldots, u_{n} \in C$, and any nonexpansive mapping $T: C \rightarrow C$, we have

$$
\left\|T\left(\sum_{j=1}^{n} \lambda_{j} u_{j}\right)-\sum_{j=1}^{n} \lambda_{j} T u_{j}\right\|^{2} \leq \sum_{i<j} \lambda_{i} \lambda_{j}\left(\left\|u_{i}-u_{j}\right\|^{2}-\left\|T u_{i}-T u_{j}\right\|^{2}\right) .
$$

Recall also that the implicit midpoint rule (IMR) [13] generates a sequence $\left\{x_{n}\right\}$ by the recursion process

$$
x_{n+1}=\left(1-t_{n}\right) x_{n}+t_{n} T\left(\frac{x_{n}+x_{n+1}}{2}\right), \quad n \geq 0,
$$

where $t_{n} \in(0,1)$ for all $n$, and $T: C \rightarrow C$ is a nonexpansive mapping.

The following properties of the IMR (2.3) are proved in [13].

Lemma 2.4 Let $\left\{t_{n}\right\}$ be any sequence in $(0,1)$ and let $\left\{x_{n}\right\}$ be the sequence generated by the $\operatorname{IMR}(2.3)$. Then

(i) $\left\|x_{n+1}-p\right\| \leq\left\|x_{n}-p\right\|$ for all $n \geq 0$ and $p \in \operatorname{Fix}(T)$. In particular, $\left\{x_{n}\right\}$ is bounded, and moreover, we have

$$
\lim _{n \rightarrow \infty}\left\|x_{n}-p\right\| \text { exists for every } p \in \operatorname{Fix}(T) .
$$

(ii) $\sum_{n=1}^{\infty} t_{n}\left\|x_{n}-x_{n+1}\right\|^{2}<\infty$.

(iii) $\sum_{n=1}^{\infty} t_{n}\left(1-t_{n}\right)\left\|x_{n}-T\left(\frac{x_{n}+x_{n+1}}{2}\right)\right\|^{2}<\infty$.

The convergence of the IMR (2.3) is proved in [13].

Theorem 2.5 Let $C$ be a nonempty closed convex subset of a Hilbert space $H$ and $T: C \rightarrow$ $C$ be a nonexpansive mapping with $\operatorname{Fix}(T) \neq \emptyset$. Assume $\left\{x_{n}\right\}$ is generated by the IMR (2.3) 
where the sequence $\left\{t_{n}\right\}$ of parameters satisfies the conditions (C1) and (C2) in the Introduction. Then $\left\{x_{n}\right\}$ converges weakly to a fixed point of $T$.

\section{Ergodicity}

In this section we discuss the ergodic convergence of the sequence $\left\{x_{n}\right\}$ generated by the IMR (2.3), that is, the convergence of the means

$$
z_{n}:=\frac{1}{s_{n}} \sum_{k=1}^{n} a_{k} x_{k}, \quad n=1,2, \ldots
$$

where $\left\{a_{n}\right\}$ is a sequence of positive numbers such that

$$
s_{n}:=\sum_{k=1}^{n} a_{k} \rightarrow \infty \quad(\text { as } n \rightarrow \infty)
$$

Set $F=\operatorname{Fix}(T)$ and let $P_{F}$ be the nearest point projection from $H$ to $F$.

Lemma 3.1 The sequence $\left\{P_{F} x_{n}\right\}$ is convergent in norm.

Proof First observe that

$$
\lim _{n \rightarrow \infty}\left\|x_{n}-P_{F} x_{n}\right\| \text { exists. }
$$

As a matter of fact, we get for $n>m$, by Lemma 2.1(i) and Lemma 2.4(i),

$$
\left\|x_{n}-P_{F} x_{n}\right\| \leq\left\|x_{n}-P_{F} x_{m}\right\| \leq\left\|x_{m}-P_{F} x_{m}\right\| .
$$

That is, $\left\{\left\|x_{n}-P_{F} x_{n}\right\|\right\}$ is decreasing and (3.3) is proven.

Applying the inequality (Lemma 2.1(iii))

$$
\left\|P_{F} v-u\right\|^{2} \leq\|v-u\|^{2}-\left\|P_{F} v-v\right\|^{2}, \quad v \in H, u \in F
$$

to the case where $v=x_{n}$ and $u=P_{F} x_{m}$ (with $n>m$ ) together with Lemma 2.4(i), we get

$$
\begin{aligned}
\left\|P_{F} x_{n}-P_{F} x_{m}\right\|^{2} & \leq\left\|x_{n}-P_{F} x_{m}\right\|^{2}-\left\|P_{F} x_{n}-x_{n}\right\|^{2} \\
& \leq\left\|x_{m}-P_{F} x_{m}\right\|^{2}-\left\|P_{F} x_{n}-x_{n}\right\|^{2} .
\end{aligned}
$$

The strong convergence of $\left\{P_{F} x_{n}\right\}$ follows immediately from the fact (3.3).

Remark 3.2 The limit of $\left\{P_{F} x_{n}\right\}$, which we denote by $\hat{p}$, can also be identified as the asymptotic center of the sequence $\left\{x_{n}\right\}$ with respect to the fixed point set $F$ of $T$. In other words,

$$
\hat{p}=\arg \min _{x \in F} f(p):=\limsup _{n \rightarrow \infty}\left\|x_{n}-p\right\|^{2} .
$$


As a matter of fact, by (3.4) we get, for any $p \in F$,

$$
\left\|P_{F} x_{n}-x_{n}\right\|^{2} \leq\left\|x_{n}-p\right\|^{2}-\left\|P_{F} x_{n}-p\right\|^{2} .
$$

Upon taking limsup we immediately obtain

$$
f(\hat{p}) \leq f(p)-\|p-\hat{p}\|^{2}, \quad p \in F .
$$

Hence, (3.5) holds.

Theorem 3.3 Let $C$ be a closed convex subset of a Hilbert space $H$ and let $T: C \rightarrow C$ be a nonexpansive mapping such that $F \equiv \operatorname{Fix}(T) \neq \emptyset$. Assume $\left\{t_{n}\right\}$ is any sequence of positive numbers in the unit interval $(0,1)$ and let $\left\{x_{n}\right\}$ be the sequence generated by the IMR $(2.3)$. Define the means $\left\{z_{n}\right\}$ by (3.1), where the weights $\left\{a_{n}\right\}$ are all positive and satisfy the condition (3.2). Assume, in addition, $\lim _{n \rightarrow \infty}\left\|x_{n}-T x_{n}\right\|=0$. Then $\left\{z_{n}\right\}$ converges weakly to a point $z$, where $z=\lim _{n \rightarrow \infty} P_{F} x_{n}$ (in norm).

Proof Let $z=\lim _{n \rightarrow \infty} P_{F} x_{n}$ which is well defined by Lemma 3.1. By Lemma 2.1(ii), we have, for each $k$,

$$
\left\langle x_{k}-P_{F} x_{k}, u-P_{F} x_{k}\right\rangle \leq 0, \quad u \in F
$$

It turns out that, for $u \in F$,

$$
\left\langle x_{k}-P_{F} x_{k}, u-z\right\rangle \leq-\left\langle x_{k}-P_{F} x_{k}, z-P_{F} x_{k}\right\rangle \leq M\left\|z-P_{F} x_{k}\right\| .
$$

(Here $M$ is a constant such that $M \geq\left\|x_{k}-P_{F} x_{k}\right\|$ for all $k$.)

By multiplying by $a_{k}$ and then summing up from $k=1$ to $n$, we conclude

$$
\left\langle z_{n}-\frac{1}{s_{n}} \sum_{k=1}^{n} a_{k} P_{F} x_{k}, u-z\right\rangle \leq \frac{M}{s_{n}} \sum_{k=1}^{n} a_{k}\left\|z-P_{F} x_{k}\right\| .
$$

We now claim that

$$
\lim _{n \rightarrow \infty}\left\|z_{n}-T z_{n}\right\|=0
$$

Consequently, by Lemma 2.2, each weak cluster point of $\left\{z_{n}\right\}$ falls in $F$.

To see (3.7), we will prove that

$$
\left\|T z_{n}-z_{n}\right\|<\delta(\varepsilon)
$$

for all $n$ big enough, where $\delta(\varepsilon) \rightarrow 0$ as $\varepsilon \rightarrow 0$. For the sake of simplicity, we may, due to the assumption $\lim _{n \rightarrow \infty}\left\|x_{n}-T x_{n}\right\|=0$, assume that

$$
\left\|x_{n}-T x_{n}\right\|<\varepsilon
$$

for all $n$. 
Let $h(t)=\sqrt{t}$ for $t \geq 0$ and let $M$ be a constant such that $M \geq 2 \cdot \operatorname{diam}\left(x_{n}\right)$. For each $n$, we put $\lambda_{j}^{(n)}=\frac{a_{j}}{s_{n}}$ for $1 \leq j \leq n$ and apply (2.2) to get

$$
\begin{aligned}
\left\|T\left(z_{n}\right)-\sum_{j=1}^{n} \lambda_{j}^{(n)} T x_{j}\right\| & \leq h\left(M \sum_{i<j} \lambda_{i}^{(n)} \lambda_{j}^{(n)}\left(\left\|x_{i}-x_{j}\right\|-\left\|T x_{i}-T x_{j}\right\|\right)\right) \\
& \leq h\left(M \sum_{i<j} \lambda_{i}^{(n)} \lambda_{j}^{(n)}\left(\left\|x_{i}-T x_{i}\right\|+\left\|x_{j}-T x_{j}\right\|\right)\right) \\
& \leq h\left(2 \varepsilon M \sum_{i<j} \lambda_{i}^{(n)} \lambda_{j}^{(n)}\right) \\
& \leq h(\varepsilon M) .
\end{aligned}
$$

Combining (3.9) and (3.10), we derive that

$$
\begin{aligned}
\left\|T\left(z_{n}\right)-z_{n}\right\| & \leq\left\|T\left(z_{n}\right)-\sum_{j=1}^{n} \lambda_{j}^{(n)} T x_{j}\right\|+\left\|\sum_{j=1}^{n} \lambda_{j}^{(n)}\left(T x_{j}-x_{j}\right)\right\| \\
& \leq h(\varepsilon M)+\sum_{j=1}^{n} \lambda_{j}^{(n)}\left\|T x_{j}-x_{j}\right\| \\
& \leq h(\varepsilon M)+\varepsilon .
\end{aligned}
$$

It turns out that (3.8) with $\delta(\varepsilon)=\sqrt{\varepsilon M}+\varepsilon$.

Now since $P_{F} x_{n} \rightarrow z$ in norm, we see that the means $\frac{1}{s_{n}} \sum_{k=1}^{n} a_{k} P_{F} x_{k} \rightarrow z$ in norm, as well. Consequently, if $\left\{z_{n_{j}}\right\}$ is a subsequence weakly converging to some point $z^{*}$, it follows from (3.6) that

$$
\left\langle z^{*}-z, u-z\right\rangle \leq 0, \quad u \in F
$$

This together with the fact that $z^{*} \in F$ implies that $z=P_{F} z^{*}=z^{*}$. That is, $z$ is the only weak cluster point of the sequence $\left\{z_{n}\right\}$ and therefore, we must have $z_{n} \rightarrow z$ weakly.

Remark 3.4 In Theorem 3.3 we assumed that $\lim _{n \rightarrow \infty}\left\|x_{n}-T x_{n}\right\|=0$. This assumption is guaranteed if the sequence $\left\{t_{n}\right\}$ satisfies the condition (C2) in the Introduction, that is, $\liminf _{n \rightarrow \infty} t_{n}>0$. Indeed, by (C2) and Lemma 2.4(ii), we find

$$
\lim _{n \rightarrow \infty}\left\|x_{n+1}-x_{n}\right\|=0
$$

Since the definition of IMR (2.3) yields

$$
\left\|x_{n+1}-x_{n}\right\|=t_{n}\left\|x_{n}-T\left(\frac{x_{n}+x_{n+1}}{2}\right)\right\|,
$$

we also have

$$
\lim _{n \rightarrow \infty}\left\|x_{n}-T\left(\frac{x_{n}+x_{n+1}}{2}\right)\right\|=0 .
$$


Combining (3.13) and (3.14), we infer that

$$
\begin{aligned}
\left\|x_{n}-T x_{n}\right\| & \leq\left\|x_{n}-T\left(\frac{x_{n}+x_{n+1}}{2}\right)\right\|+\left\|T x_{n}-T\left(\frac{x_{n}+x_{n+1}}{2}\right)\right\| \\
& \leq\left\|x_{n}-T\left(\frac{x_{n}+x_{n+1}}{2}\right)\right\|+\frac{1}{2}\left\|x_{n}-x_{n+1}\right\| \rightarrow 0 .
\end{aligned}
$$

Remark 3.5 If we assume (3.9) holds for all $n>N$, then we need some more delicate technicalities dealing with (3.10). We may proceed as follows. Decompose $z_{n}($ for $n>N)$ as

$$
z_{n}=\frac{s_{N}}{s_{n}} z_{N}+\frac{s_{n}^{N}}{s_{n}} z_{n}^{N}
$$

where

$$
s_{n}^{N}=\sum_{j=N+1}^{n} a_{j}, \quad z_{n}^{N}=\sum_{j=N+1}^{n} \lambda_{j}^{(n, N)} x_{j}, \quad \lambda_{j}^{(n, N)}=\frac{a_{j}}{s_{n}^{N}}, \quad n>N .
$$

As $s_{n} \rightarrow \infty$, we may assume $\frac{s_{N}}{s_{n}}\left\|z_{N}\right\|<\varepsilon$. Repeating the argument for (3.10) and (3.11), we get

$$
\left\|T\left(z_{n}^{N}\right)-z_{n}^{N}\right\| \leq h(\varepsilon M)+\varepsilon
$$

Let $M_{1}=\sup _{n \geq 0}\left\|x_{n}\right\|$. We finally obtain, for $n>N$,

$$
\begin{aligned}
\left\|T\left(z_{n}\right)-z_{n}\right\| & \leq\left\|T\left(z_{n}\right)-T\left(z_{n}^{N}\right)\right\|+\left\|T\left(z_{n}^{N}\right)-z_{n}^{N}\right\|+\left\|z_{n}^{N}-z_{n}\right\| \\
& \leq\left\|T\left(z_{n}^{N}\right)-z_{n}^{N}\right\|+2\left\|z_{n}^{N}-z_{n}\right\| \\
& =\left\|T\left(z_{n}^{N}\right)-z_{n}^{N}\right\|+2 \frac{s_{N}}{s_{n}}\left\|z_{N}-z_{n}^{N}\right\| \\
& \leq h(\varepsilon M)+\varepsilon+4 M_{1} \varepsilon .
\end{aligned}
$$

Next we show that in some circumstances, the sequence $\left\{z_{n}\right\}$ can converge strongly.

Theorem 3.6 Let the assumptions of Theorem 3.3 holds. Then the sequence $\left\{z_{n}\right\}$ converges in norm to the point $z=\lim _{n \rightarrow \infty} P_{F} x_{n}$ if, in addition, any one of the following conditions is satisfied:

(i) The fixed point set $F$ of $T$ has nonempty interior.

(ii) $T$ is a contraction, that is,

$$
\|T x-T y\| \leq \rho\|x-y\|, \quad x, y \in C,
$$

where $\rho \in[0,1)$ is a constant. In this case, the sequence $\left\{x_{n}\right\}$ generated by the IMR (2.3) converges in norm to the unique fixed point of $T$.

(iii) $T$ is compact, namely, $T$ maps bounded sets to relatively norm-compact sets.

Proof (i) By assumption, we have $x_{0} \in F$ and $\delta>0$ such that

- $x_{0}+\delta w \in F$ for all $w \in H$ such that $\|w\| \leq 1$. 
Therefore, upon substituting $x_{0}+\delta w$ for $u$ in (3.6) we obtain

$$
\begin{aligned}
& \delta\left\langle z_{n}-\frac{1}{s_{n}} \sum_{k=1}^{n} a_{k} P_{F} x_{k}, w\right\rangle \\
& \leq-\left\langle z_{n}-\frac{1}{s_{n}} \sum_{k=1}^{n} a_{k} P_{F} x_{k}, x_{0}-z\right\rangle+\frac{M}{s_{n}} \sum_{k=1}^{n} a_{k}\left\|z-P_{F} x_{k}\right\|,
\end{aligned}
$$

for all $w \in H$ such that $\|w\| \leq 1$.

Taking the supremum in (3.15) over $w \in H$ such that $\|w\| \leq 1$ immediately yields

$$
\begin{aligned}
\delta \| & z_{n}-\frac{1}{s_{n}} \sum_{k=1}^{n} a_{k} P_{F} x_{k} \| \\
& \leq-\left\langle z_{n}-\frac{1}{s_{n}} \sum_{k=1}^{n} a_{k} P_{F} x_{k}, x_{0}-z\right\rangle+\frac{M}{s_{n}} \sum_{k=1}^{n} a_{k}\left\|z-P_{F} x_{k}\right\| \rightarrow 0 .
\end{aligned}
$$

This verifies that $z_{n} \rightarrow z$ in norm.

(ii) Since $T$ is a contraction, $T$ has a unique fixed point which is denoted by $p$. By (2.3) we deduce that (noticing $\left\|x_{n+1}-p\right\| \leq\left\|x_{n}-p\right\|$ )

$$
\begin{aligned}
\left\|x_{n+1}-p\right\| & \leq\left(1-t_{n}\right)\left\|x_{n}-p\right\|+t_{n} \rho\left\|\frac{1}{2}\left(x_{n}+x_{n+1}\right)-p\right\| \\
& \leq\left(1-t_{n}\right)\left\|x_{n}-p\right\|+\frac{1}{2} \rho t_{n}\left(\left\|x_{n}-p\right\|+\left\|x_{n+1}-p\right\|\right) \\
& \leq\left(1-(1-\rho) t_{n}\right)\left\|x_{n}-p\right\| .
\end{aligned}
$$

It turns out that

$$
(1-\rho) t_{n}\left\|x_{n}-p\right\| \leq\left\|x_{n}-p\right\|-\left\|x_{n+1}-p\right\|
$$

and hence

$$
\sum_{n=1}^{\infty} t_{n}\left\|x_{n}-p\right\|<\infty
$$

Since $\sum_{n=1}^{\infty} t_{n}=\infty$, we must have $\liminf _{n \rightarrow \infty}\left\|x_{n}-p\right\|=0$. However, since the sequence $\left\{\left\|x_{n}-p\right\|\right\}$ is decreasing, we must have $\lim _{n \rightarrow \infty}\left\|x_{n}-p\right\|=0$. Namely, $x_{n} \rightarrow p$ in norm, and so $z_{n} \rightarrow p$ in norm.

(iii) Since $T$ is compact and since $\left\{z_{n}\right\}$ is weakly convergent, $\left\{T z_{n}\right\}$ is relatively normcompact. This together with (3.7) evidently implies that $\left\{z_{n}\right\}$ is relatively norm-compact. Therefore, $\left\{z_{n}\right\}$ must converge in norm to $z=\lim _{n \rightarrow \infty} P_{F} x_{n}$. 


\section{Author details}

${ }^{1}$ Department of Mathematics, School of Science, Hangzhou Dianzi University, Hangzhou, Zhejiang 310018, China.

${ }^{2}$ Department of Mathematics, King Abdulaziz University, P.O. Box 80203, Jeddah, 21589, Saudi Arabia. ${ }^{3}$ Department of Mathematics, Sciences Faculty for Girls, King Abdulaziz University, P.O. Box 4087, Jeddah, 21491, Saudi Arabia.

\section{Acknowledgements}

The authors are grateful to the anonymous referees for their helpful comments and suggestions, which improved the presentation of this manuscript. This project was funded by the Deanship of Scientific Research (DSR), King Abdulaziz University, under grant No. (49-130-35-HiCi). The authors, therefore, acknowledge technical and financial support of KAU.

\section{Received: 24 October 2014 Accepted: 8 December 2014 Published: 06 Jan 2015}

\section{References}

1. Baillon, J-B: Un théorème de type ergodique pour les contractions non linéaires dans un espace de Hilbert. C. R. Acad. Sci. Paris Sér. A-B 280(22), Aii, A1511-A1514 (1975) (in French)

2. Bruck, RE: A simple proof of the mean ergodic theorem for nonlinear contractions in Banach spaces. Isr. J. Math. 32(2-3), 107-116 (1979)

3. Baillon, J-B, Clement, P: Ergodic theorems for nonlinear Volterra equations in Hilbert space. Nonlinear Anal. 5(7), 789-801 (1981)

4. Passty, GB: Ergodic convergence to a zero of the sum of monotone operators in Hilbert spaces. J. Math. Anal. Appl. 72, 383-390 (1979)

5. Lions, PL, Mercier, B: Splitting algorithms for the sum of two nonlinear operators. SIAM J. Numer. Anal. 16, 964-979 (1979)

6. Auzinger, W, Frank, R: Asymptotic error expansions for stiff equations: an analysis for the implicit midpoint and trapezoidal rules in the strongly stiff case. Numer. Math. 56, 469-499 (1989)

7. Bader, G, Deuflhard, P: A semi-implicit mid-point rule for stiff systems of ordinary differential equations. Numer. Math 41, 373-398 (1983)

8. Deuflhard, P: Recent progress in extrapolation methods for ordinary differential equations. SIAM Rev. 27(4), 505-535 (1985)

9. Edith, E: Numerical and approximative methods in some mathematical models. Ph.D. thesis, Babes-Bolyai University of Cluj-Napoca (2006)

10. Schneider, C: Analysis of the linearly implicit mid-point rule for differential-algebra equations. Electron. Trans. Numer. Anal. 1, 1-10 (1993)

11. Somalia, S: Implicit midpoint rule to the nonlinear degenerate boundary value problems. Int. J. Comput. Math. 79(3), 327-332 (2002)

12. Somalia, S, Davulcua, S: Implicit midpoint rule and extrapolation to singularly perturbed boundary value problems. Int. J. Comput. Math. 75(1), 117-127 (2000)

13. Alghamdi, MA, Alghamdi, MA, Shahzad, N, Xu, HK: The implicit midpoint rule for nonexpansive mappings. Fixed Point Theory Appl. 2014, 96 (2014)

14. Goebel, K, Kirk, WA: Topics in Metric Fixed Point Theory. Cambridge Studies in Advanced Mathematics, vol. 28. Cambridge University Press, Cambridge (1990)

15. Zarantonello, EH: Projections on convex sets in Hilbert space and spectral theory. In: Zarantonello, EH (ed.) Contributions to Nonlinear Functional Analysis, pp. 237-424. Academic Press, New York (1971)

10.1186/1029-242X-2015-4

Cite this article as: Xu et al.: Ergodicity of the implicit midpoint rule for nonexpansive mappings. Journal of Inequalities and Applications 2015, 2015:4

\section{Submit your manuscript to a SpringerOpen ${ }^{\circ}$ journal and benefit from:}

- Convenient online submission

- Rigorous peer review

Immediate publication on acceptance

- Open access: articles freely available online

- High visibility within the field

- Retaining the copyright to your article 